\title{
Tratamento térmico de frutos da cajazeira utilizando vapor d'água, visando ao controle de Ceratitis capitata e à qualidade do fruto
}

\author{
Thermal treatment of Spondias mombin fruits using water vapour obsectiving control of Ceratitis \\ capitata and fruit quality
}

\author{
Carlos Henrique de Brito ${ }^{\mathrm{I}^{*}}$ Nivânia Pereira da Costa ${ }^{\mathrm{I}}$ Jacinto de Luna Batista $^{\mathrm{I}}$ \\ Aldeni Barbosa da Silva ${ }^{I}$ Antônio Nustenil de Lima ${ }^{I}$
}

\section{RESUMO}

As moscas-das-frutas atacam, preferencialmente, frutos maduros, mas podem atacar frutos verdes, nos quais depositam seus ovos. O fruto atacado fica mole e sua polpa totalmente comprometida. $O$ objetivo deste trabalho foi avaliar a eficiência do tratamento térmico com vapor visando a controlar C. capitata sem danificar a qualidade do fruto de cajá. Inicialmente, os frutos foram colhidos, desinfestados e padronizados em função do tamanho e do ponto de maturação. Em seguida, foram seccionados longitudinalmente e infestados com dez ovos/fruto com idade de 24 horas. Após 72 horas da infestação, os frutos foram submetidos ao tratamento com vapor nas temperaturas de 46 e $50^{\circ} \mathrm{C}$, nos tempos de exposição zero, dez, 20, 30 e 40 minutos. As avaliações de mortalidade foram realizadas após 7 dias, através da contagem do número de larvas sobreviventes. A análise química da polpa, quanto ao teor de sólidos solúveis totais, acidez total titulável e $\mathrm{pH}$ foi realizada com 48 horas após o tratamento térmico. Na temperatura de $46^{\circ} \mathrm{C}$, os frutos de cajá devem permanecer expostos ao vapor pelo tempo de 27 minutos e, na temperatura de $50^{\circ} \mathrm{C}$, por 20 minutos, visando ao controle de larvas de $\boldsymbol{C}$. capitata, sem comprometimento das características físico-químicas avaliadas.

Palavras-chave: mosca do Mediterrâneo, Spondias monbin, tratamento térmico, pós-colheita.

\section{ABSTRACT}

Fruit-flies attack mature fruits preferentially, and they could also attack immature fruits, and deposit its eggs. Fruits become soft and injured and pulp is totally compromised. The objective of this study was to evaluate efficiency of thermal treatment with vapor aiming C. capitata control without damaging fruit Spondias mombin quality. First, fruits had been harvested, disinfested and standardized according of the size and maturation point. After that, fruits were seccioned longitudinally and infested with ten eggs/fruits at 24-hour age.
After 72 hours infestation, fruits were submitted at treatment with vapour at 46 and $50^{\circ} \mathrm{C}$ in exposition times of zero, ten, 20, 30 and 40 minutes. Mortality evaluations had been carried through 7 days after, by surviving larvae counting. Chemical pulp analysis, was done based on the total of soluble solids, titrable acidity and the $\mathrm{pH}$, the thermal treatment, was carried 48 hours after. The temperature of $46^{\circ} \mathrm{C}, \boldsymbol{S}$. mombin fruits must remain displayed on vapour for 27 minutes and at $50^{\circ} \mathrm{C}$ during 20 minutes, aiming the control of the C. capitata larvae fruits flies, without compromising the characteristics physico-chemistry evaluated.

Key words: mediterranean fruit fly, Spondias monbin, thermal treatment, postharvest.

\section{INTRODUÇÃO}

As moscas-das-frutas fazem parte de um grupo de pragas responsáveis por grandes prejuízos econômicos, não só pelos danos diretos que causam à produção, como também pelas barreiras quarentenárias impostas pelos países importadores. Os danos das moscas-das-frutas são causados diretamente nos frutos pela fêmea adulta (perfuração do fruto por ocasião da oviposição) e pelas larvas (consumo da polpa provocando um apodrecimento interno) (SOUZA FILHO et al., 2004).

No Brasil, as espécies de moscas-das-frutas de larga distribuição geográfica pertencem aos gêneros Anastrepha e Ceratitis. Ceratitis capitata (Wied.), é uma praga exótica, conhecida também como moscado-mediterrâneo, é a única espécie do gênero registrada no Brasil, onde está amplamente disseminada e

IDepartamento de Fitotecnia, Universidade Federal da Paraíba (UFPB), 58397-000, Areia, PB, Brasil. E-mail: chbrito1@hotmail.com. *Autor para correspondência. 
adaptada a ambientes rurais e urbanos (MONTES \& RAGA, 2006). A mosca-do-mediterrâneo é a mais importante mosca-da-fruta do ponto de vista de dano econômico à fruticultura mundial, sendo a espécie de Tephritidae mais cosmopolita (MALAVASI et al., 2000).

É uma espécie altamente colonizadora em relação a outras que têm distribuição restrita e baixa capacidade de se adaptarem a novos ambientes (MALAVASI, 2001). No Brasil, é encontrada desde o Estado do Rio Grande do Sul até alguns Estados do norte e nordeste.

O Brasil é o terceiro maior produtor de frutas frescas do mundo, produz cerca de 35 milhões de toneladas anualmente e gera cerca de 4 milhões de empregos diretos, sendo que o nordeste brasileiro destaca-se como um dos maiores produtores e exportadores de frutas do país (AGRIANUAL, 2005).

A cajazeira (Spondias mombin L.), planta da família Anacardeaceae, situa-se entre as frutíferas que se adaptam muito bem às condições nordestinas, produzindo, embora ainda de forma silvestre, frutos nutritivos, saborosos e de grande aceitação pelo mercado consumidor nacional (BOSCO et al., 2000). Os frutos são comercializados e podem ser consumidos "in natura” ou na forma de sucos, sorvetes, picolés e cremes (QUEIROZ, 2000; AZEVEDO et al., 2004).

Dentre as espécies de fruteiras infestadas por C. capitata, o cajá é bastante apreciado e muito bem adaptado às condições edafoclimáticas da Região Nordeste. $\mathrm{O}$ ataque das moscas-das-frutas se inicia, quando o fruto se encontra verde-maturo, que é caracterizado por apresentar em uma das extremidades um tom ligeiramente esverdeado e o restante da casca com coloração amarelo-clara (COSTA, 1998). Os ovos são depositados no interior dos frutos e, após a eclosão, as larvas se alimentam da polpa e facilitam a entrada de organismos saprófitos, como fungos e bactérias bem como provocam a podridão e queda dos frutos sendo recomendado, inicialmente, monitoramento no campo e, posteriormente controle, que pode ser por métodos físicos ou biológicos (VIEIRA NETO, 2002). As perdas diretas podem ser observadas pela diminuição da produção, aumento do custo de produção, menor valor da produção e menor vida útil. Frutas produzidas em áreas infestadas não podem ser exportadas para países com barreiras quarentenárias (MALAVASI, 2001).

Na Região Nordeste, onde existem poucos plantios comerciais é consumida em toda a região e, ultimamente, é exportada para outras regiões do país, na forma de polpa, nas quais já existe um mercado consumidor assegurado, pois esse produto é largamente utilizado na forma de suco e sorvete. $\mathrm{O}$ Brasil, por possuir um extenso território, destaca-se com um percentual significativo no volume e produção mundial de frutas, no entanto, são insignificantes as quantidades de frutas exóticas nativas da Região Nordeste que são exportadas, como o cajá (S. mombin L.), umbu (S. tuberosa Arruda Câmara), ceriguela ( $\boldsymbol{S}$. purpurea L.), entre outras (SILVA et al., 2002).

Dentre os tratamentos físicos realizados na pós-colheita, aquele utilizando ar quente saturado com vapor de água e temperaturas variando entre 40 a $50^{\circ} \mathrm{C}$ foi utilizado com sucesso na redução da infestação dos frutos com $\boldsymbol{C}$. capitata em manga cv. 'Tommy Atkins' (BATISTA et al., 2005), goiaba, Psidium guajava (DÓRIA et al., 2001, 2004), e sapoti, Achras sapota, (LIMA, 2005). Esse método foi desenvolvido na Florida, em 1929, para evitar a dispersão de $\boldsymbol{C}$. capitata (BAKER et al., 1990). No entanto, é necessário avaliar qual a faixa de temperatura e tempo de exposição mais adequados, que garantam a manutenção da qualidade intrínseca e ampliação da vida útil póscolheita do fruto.

O objetivo deste trabalho foi avaliar a eficiência do tratamento, utilizando vapor d'agua para frutos da cajazeira, visando a controlar $C$. capitata sem causar prejuízo à qualidade do fruto.

\section{MATERIAL E MÉTODOS}

A pesquisa foi desenvolvida com frutos da cajazeira, selecionados de três plantas, pertencentes ao Centro de Ciências Agrárias - CCA da Universidade Federal da Paraíba (UFPB), Areia PB. Os frutos foram colhidos com auxílio de um podão e uma rede coletora estendida a $15 \mathrm{~cm}$ do solo, para evitar os danos mecânicos ocasionados pelo impacto da queda. Por ocasião da colheita, os frutos foram acondicionados em caixas plásticas $(60,0 \mathrm{~cm}$ x 80,0cm x $30,0 \mathrm{~cm}$ ) e padronizados em função do tamanho (comprimento médio de $36,8 \mathrm{~mm}$ ) e estádio de maturação, de acordo com caracterização descrita por COSTA (1998). No Laboratório de Entomologia do Departamento de Fitotecnia/UFPB, os frutos foram submetidos ao tratamento de imersão em solução com 50,0ppm de hipoclorito de sódio por cinco minutos, sendo em seguida colocados para secar sobre papel toalha. A infestação dos frutos foi realizada com ovos de $\boldsymbol{C}$. capitata, obtidos da criação mantida no laboratório de entomologia. Realizou-se um corte longitudinal de $1,0 \mathrm{~cm}$ no fruto onde foram colocados dez ovos/fruto com idade de 24 horas, com auxílio de pincel $n^{\circ} 1$ (DÓRIA et al., 2004). Os cortes foram vedados com fita crepe e os frutos colocados em bandejas plásticas e mantidos em prateleiras sob condições não controladas. 
Decorridas 72 horas da infestação, os frutos foram submetidos a um ambiente saturado de vapor d'agua, nas temperaturas de 46 e $50^{\circ} \mathrm{C}$, nos tempos de exposição zero (testemunha), dez, 20, 30 e 40 minutos, com base em metodologia de BATISTA (2001). De acordo com esse autor, utilizou-se uma câmara de vaporização adaptada em uma estufa, medindo 72 x 63 $\mathrm{x} 48 \mathrm{~cm}$ e, para a circulação do vapor no interior da câmara, foi colocado, na extremidade lateral inferior, um ventilador com 2500rpm. A temperatura foi controlada com o auxílio de um termômetro colocado no mesmo nível da prateleira, em que se encontravam os frutos a serem tratados e monitorados por um termosensor fixado na câmara. O vapor foi injetado na câmara por meio de vaporizador da marca Delonghi, com 1400W e 3,5bar, conectado na base inferior da câmara. As avaliações de mortalidade foram realizadas após sete dias do tratamento, pela contagem do número de larvas sobreviventes.

A avaliação físico-química da polpa foi realizada 48 horas após o tratamento térmico, quantificando-se o teor de sólidos solúveis totais (SST), acidez total titulável (ATT) e pH, conforme indicação da AOAC (1992).

Para a análise de mortalidade, o delineamento experimental foi o inteiramente casualizado, em arranjo fatorial $2 \times 4+1$ (duas temperaturas, quatro tempos de exposição ao vapor + testemunha), onde cada tratamento constitui-se de cinco repetições de dez frutos. Os fatores qualitativos foram comparados pelo teste qui-quadrado e o quantitativo analisado por Probit. O delineamento utilizado para avaliar as análises químicas foi o DIC, no qual cada tratamento constituise de três repetições de dez frutos, sendo as médias comparadas pelo teste de Tukey a 5\% de probabilidade. Todas as análises estatísticas foram realizadas utilizando o software SAEG, versão 9,0 (SAEG, 2005).

\section{RESULTADOS E DISCUSSÃO}

Constatou-se diferença significativa entre as temperaturas de 46 e $50^{\circ} \mathrm{C}$ sob a mortalidade de ovos e/ou larvas de $\boldsymbol{C}$. capitata (Tabela 1). Nos tempos de exposição de dez, 20 e 30 minutos, na temperatura de $46^{\circ} \mathrm{C}$, a mortalidade foi sempre inferior, quando comparada com a temperatura de $50^{\circ} \mathrm{C}$, não havendo diferença para o tempo de 40 minutos de exposição.

Na figura 1, ilustra-se o resultado da análise de Probit, indicando a probabilidade de morte de $\boldsymbol{C}$. capitata para cada temperatura testada $\left(46\right.$ e $\left.50^{\circ} \mathrm{C}\right)$ e para cada tempo de exposição (zero, dez, 20, 30 e 40 minutos). O Probit é originário do cálculo entre o número de ovos colocados nos frutos, estes submetidos ao
Tabela 1 - Porcentagem de ovos e larvas mortas de Ceratitis capitata em frutos da cajazeira, submetidos ao tratamento com vapor a duas temperaturas e diferentes tempos de exposição. CCA/UFPB, Areia, 2005.

\begin{tabular}{lll}
\hline Tratamentos & $46^{\circ} \mathrm{C}$ & $50^{\circ} \mathrm{C}$ \\
\hline Testemunha & $4,2 \mathrm{~A}$ & $4,2 \mathrm{~A}$ \\
Vapor 10' & $58,6 \mathrm{~B}$ & $73,0 \mathrm{~A}$ \\
Vapor 20' & $81,0 \mathrm{~B}$ & $91,0 \mathrm{~A}$ \\
Vapor 30' & $95,7 \mathrm{~B}$ & $100,0 \mathrm{~A}$ \\
Vapor 40' & $100,0 \mathrm{~A}$ & $100,0 \mathrm{~A}$ \\
\hline
\end{tabular}

Médias não seguidas pela mesma letra maiúscula na linha diferem entre si pelo teste de qui-quadrado a $5 \%$ de probabilidade de erro.

tratamento térmico com vapor, e a sobrevivência dos ovos e/ou larvas após o tratamento.

A probabilidade de morte variou pouco mais de $40 \%$ até $100 \%$, em função do tempo de exposição para ambas as temperaturas (Figura 1). Para a temperatura de $46^{\circ} \mathrm{C}$, o tempo de exposição de 27 minutos foi suficiente para atingir o Probit 9, estimado pelo modelo e, na temperatura de $50^{\circ} \mathrm{C}$, o tempo de exposição necessário foi apenas de 20 minutos.

O objetivo no tratamento quarentenário para mosca-das-frutas, é alcançar uma mortalidade de ovos e/ou larvas de 99,9968\% (Probit 9), exigida pelo Departamento de Agricultura dos Estados Unidos USDA (BAKER, 1939 Citado por MENDONÇA et al. 2000). Em termos quarentenários, isso equivale a três sobreviventes numa população inicial de 100.000 insetos. Esse nível de exigência baseia-se no pressuposto de que, na pré-colheita, o nível de infestação é extremamente alto (NASCIMENTO \& MENDONÇA, 1998).

Utilizando o vapor a $50^{\circ} \mathrm{C}$ para tratamento de manga infestadas com $\boldsymbol{C}$. capitata, BATISTA et al. (2005) estimaram que o tempo de 90 minutos foi eficiente para o tratamento quarentenário dessa espécie de inseto. GAFFNEY et al. (1990) utilizaram vapor quente a $46,1^{\circ} \mathrm{C}$ em frutos de manga e, $43,3^{\circ} \mathrm{C}$ para "grapefruit", visando o controle de ovos e larvas de mosca-dasfrutas e verificaram redução, no período de exposição ao vapor, para 3-4 horas, comparado a tratamentos anteriormente realizados com vapor.

Apesar dos tratamentos térmicos apresentarem resultados satisfatórios no controle de moscas-das-frutas na pós-colheita, faz-se necessário avaliar seus efeitos sob a qualidade intrínseca dos frutos.

Na avaliação da qualidade, utilizaram-se somente os frutos submetidos à temperatura de $50^{\circ} \mathrm{C}$, os quais apresentaram, após 48 horas, os valores de 


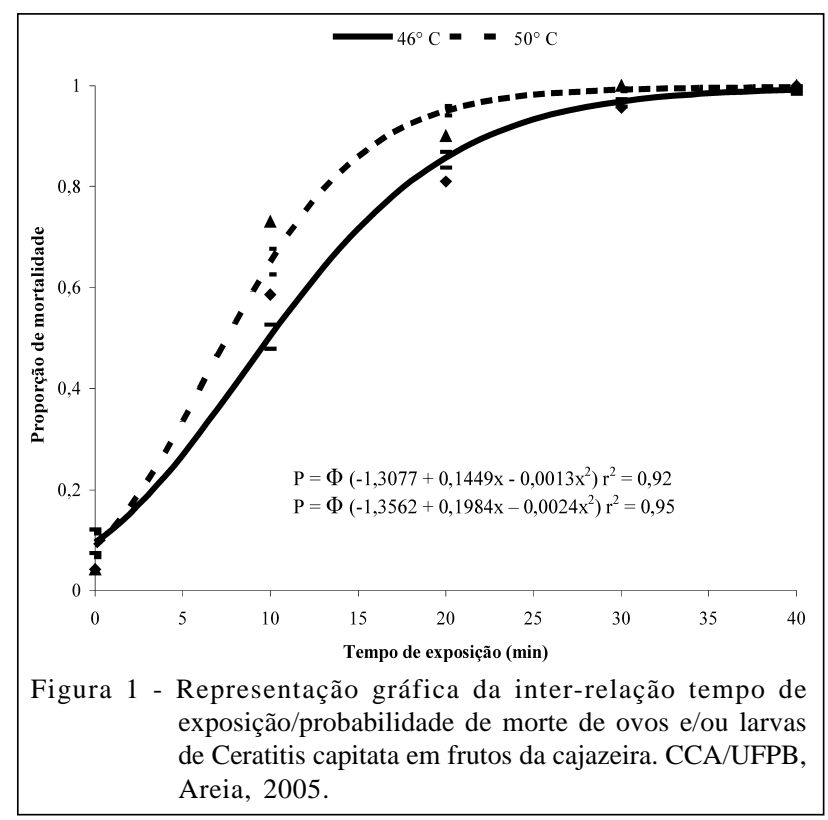

sólidos solúveis totais (SST), pH e acidez total titulável descritos na tabela 2.

Os valores médios de sólidos solúveis totais (SST) variaram, significativamente, na faixa de 9,66 a 11,80 Brix , sendo a média $10,73^{\circ}$ Brix acima do mínimo exigido $\left(9,00^{\circ}\right.$ Brix) pelo Padrão de Identidade e Qualidade (PIQ) para polpa de cajá (BRASIL, 2000). O menor valor foi encontrado para frutos submetidos ao tratamento com vapor 40 minutos e o maior para testemunha. Observou-se que houve um pequeno decréscimo para valores de SST, à medida que aumentava o tempo de exposição dos frutos. Esse comportamento pode ser decorrente da variação de maturação entre os frutos utilizados no experimento. Nesse caso, assim como para os demais parâmetros,

Tabela 2 - Efeito do vapor no teor de sólidos solúveis (SST), pH e acidez total titulável (ATT - \% de acido cítrico) de frutos da cajazeira, após 48 horas de aplicação do trata mento. CCA/UFPB, A reia, 2005.

\begin{tabular}{llll}
\hline Tratamentos & SST (Brix) & \multicolumn{1}{c}{ pH } & ATT \\
\cline { 3 - 4 } Testemunha & $11,80 \mathrm{a}$ & $2,56 \mathrm{c}$ & $2,08 \mathrm{a}$ \\
Vapor 10' & $11,30 \mathrm{ab}$ & $2,61 \mathrm{~b}$ & $1,88 \mathrm{~b}$ \\
Vapor 20' & $10,70 \mathrm{~b}$ & $2,61 \mathrm{~b}$ & $1,93 \mathrm{~b}$ \\
Vapor 30' & $10,86 \mathrm{~b}$ & $2,59 \mathrm{bc}$ & $1,92 \mathrm{~b}$ \\
Vapor 40, & $9,66 \mathrm{c}$ & $2,68 \mathrm{a}$ & $1,86 \mathrm{~b}$ \\
C.V. (\%) & 2,87 & 0,99 & 2,78 \\
Média geral & 10,86 & 2,61 & 1,93 \\
D.M.S. & 0,83 & 0,04 & 0,14 \\
\hline
\end{tabular}

Médias não seguidas pela mesma letra minúscula na coluna diferem entre si pelo teste de Tukey a $5 \%$ de probabilidade de erro. para avaliar com mais clareza o efeito do tratamento térmico sobre os SST, seria necessário que os frutos fossem avaliados por um período de tempo mais longo, visto que esta avaliação foi realizada apenas após 48h da aplicação do tratamento térmico. No entanto, a média $\left(10,73^{\circ} \mathrm{Brix}\right)$ foi superior às relatadas por OLIVEIRA et al. (2000) e BASTOS et al. (1999) que obtiveram valores de

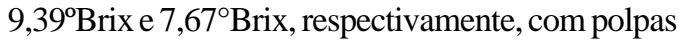
congeladas, porém, inferior à descrita por SILVA et al. (1997), 11,19Brix, com suco polposo de cajá. Para o $\mathrm{pH}$, encontraram-se valores com variações na faixa de 2,56 a 2,68, com média de 2,62 (Tabela 2). Os menores valores de $\mathrm{pH}$ foram encontrados para o tratamento testemunha e os maiores valores foram para frutos tratados com vapor 40 minutos. Esses valores estão acima dos valores mínimos do PIQ para polpa de cajá, que é de 2,2 (BRASIL, 2000). A média observada foi superior às encontradas, em polpa congelada, por OLIVEIRA et al. (1999) (2,57) e BASTOS et al. (1999) $(2,54)$, e inferiores às apresentadas por OLIVEIRA et al. (2000), que foi de 2,81, em polpas congeladas, no Estado da Bahia, e por SILVA et al. (1997) cujo pH foi de 2,78 em suco polposo. Os dados confirmam ser a polpa de cajá bastante ácida. Os resultados obtidos são considerados satisfatórios, segundo padrão exigido para polpa de cajá.

Com relação à acidez total titulável encontraram-se variações na faixa de 1,86 a 2,08\% de acido cítrico, com média de 1,97\% (Tabela 2). O menor valor de acidez total foi encontrado no tratamento vapor 40 minutos e o maior valor foi para o tratamento testemunha. Para todos os tratamentos, os níveis de ATT apresentaram-se acima do mínimo (0,90\%) exigido pelo PIQ (BRASIL, 2000). O valor médio de ATT, neste trabalho, está acima do encontrado por SILVA et al. (1999) com polpa in natura $(1,43 \%)$ e por BASTOS et al. (1999) e OLIVEIRA et al. (2000) com polpa congelada $(1,12 \%$ e $1,20 \%)$, respectivamente. De acordo com SILVA (1995), o pH em frutos de cajá é de, aproximadamente, 2,57; acidez total titulável de 1,65\% de ácido cítrico e os SSTs 10,48\%.

\section{CONCLUSÕES}

Os frutos de cajá devem permanecer expostos ao vapor de água pelo tempo de 27 minutos na temperatura de $46^{\circ} \mathrm{C}$, e na temperatura de $50^{\circ} \mathrm{C}$, apenas por 20 minutos, visando o controle de Ceratitis capitata, sem comprometimento das características físico-químicas avaliadas. 


\section{REFERÊNCIAS}

AGRIANUAL: Anuário da agricultura brasileira. São Paulo: FNP Consultoria \& Comércio, 2005. p.314-318.

AOAC. Official methods analysis of the association of official analytical chemistry. Washington, DC, 1992. p.1015

AZEVEDO, D.M. et al. Característica da germinação e morfologia do endocarpo e plântula de taperebá (Spondias mombin L.) - Anarcadiaceae. Revista Brasileira de Fruticultura, Jaboticabal, v.26, n.3, p.534-537, 2004.

BAKER, A.C. The basis for treatment of products where fruit flies are involved as a condition for entry into the United States. Florida: USDA, 1939. 8p. (Circ. $\mathrm{n}^{\circ}$ 551).

BAKER, F.T. et al. Development of a maximum pest limit for fruit flies (Diptera: Tephritidae) in produce imported into New Zealand. Journal of Economic Entomology, v.83, n.3, p.1317, 1990.

BASTOS, M. do S.R. et. al. Análise qualitativa e tecnológica da agroindústria de polpa de fruta na região Nordeste. Revista Brasileira de Fruticultura, v.21, n.3, p.252-257, 1999.

BATISTA, J.L. Influência do tratamento térmico com água e vapor na mortalidade das fases imaturas de Ceratitis capitata (Diptera: Tephritidae) e qualidade dos frutos de manga cv. Tommy Atkins. 2001. 85f. Tese (Doutorado em Produção Vegetal) - Faculdade de Ciências Agrárias e Veterinárias, Universidade Estadual Paulista.

BATISTA, J.L. et al. Efeito do tratamento com vapor quente na mortalidade de mosca-das-frutas (Ceratitis capitata) e na qualidade pós- colheita de frutos de manga (Mangifera indica L.) cv. 'Tommy Atkins'. Revista de Ciências Agrárias n.43, p.83-90, 2005

BOSCO, J. et al. A cultura da cajazeira. João Pessoa: EMEPA -PB, 2000. 229p. (Documentos, 28).

BRASIL. Ministério da Agricultura, Pecuária e Abastecimento Instrução Normativa $n^{0} 1$, de 7 de janeiro de 2000. Aprovar 0 regulamento técnico geral para a fixação de padrões de identidade e qualidade para polpa de fruta. Diário Oficial da União, Brasília, 10 de jan. 2000. Seção 1, p.54.

COSTA, N.P. Desenvolvimento, maturação e conservação pós-colheita de frutos da cajazeira (Spondias mombin L.) 1998. 98f. Dissertação (Mestrado em Produção Vegetal) Centro de Ciências Agrárias, Universidade Federal da Paraíba.

DÓRIA, H.O.S. et al. A. Efeito do tratamento com vapor quente na mortalidade de larvas de mosca-das-frutas (Anatrepha spp.) e na qualidade de goiabas (Psidium guajava L.). Revista Brasileira de Fruticultura, Jaboticabal, v.23, n.1, p.190192, 2001.

DÓRIA, H.O.S. et al. Influência de tratamentos térmicos na eliminação de Ceratitis capitata em frutos de goiaba (Psidium guajava L.). Acta Scientiarum, v.26, n.1, p.107-111, 2004.

GAFFNEY, J.J. et al. Vapor heat research unit for insect quarantine treatments. Journal of Economic Entomology, v.83, n.5, p.1965-1971, 1990 .

LIMA, A.N. Uso do tratamento térmico no controle de Ceratitis capitata em frutos de sapotizeiro (Achras sapota L.). 2005. 140f. Tese (Doutorado em Agronomia) - Centro de Ciências Agrárias, Universidade Federal da Paraíba, Areia.
MALAVASI, A. et al. Biogeografia. In: MALAVASI, A.; ZUCCHI, R.A. (Eds.). Moscas-dasfrutas de importância econômica no Brasil - conhecimento básico e aplicado. Ribeirão Preto: Holos Editora, 2000. p.41-48.

MALAVASI, A. Mosca-da-carambola, Bactrocera carambolae (Diptera: Tephretidae). In: VILELA, E.F. et al. (Ed.). Histórico e impacto de pragas introduzidas no Brasil. Ribeirão Preto: Holos, 2001. p.39-41.

MENDONÇA, M.C. et al. A. Efeito do tratamento hidrotérmico de mangas na mortalidade de larvas de Ceratitis capitata (Wied.) (Diptera: Tephritidae). Anais da Sociedade Entomológica do Brasil, v.29, n.1, p.139145, 2000.

MONTES, S.M.N.M.; RAGA, A. Eficácia de atrativos para monitoramento de Ceratitis capitata (Diptera: Tephritidae) em pomar de citrus. Arquivo do Instituto Biológico, v.73, n.3, p.317-323, 2006

NASCIMENTO, A.S.; MENDONÇA, M.C. Tratamento hidrotérmico de manga visando ao controle de moscasdas-frutas. Cruz das Almas: BA: EMBRAPA-CNPMF, 1998. 17p. (Boletim de Pesquisa, 13)

OLIVEIRA, M.E.B. et al. Avaliação de parâmetros de qualidade físico-químicos de polpas congeladas de acerola, cajá e caju. Ciência e Tecnologia de Alimentos, v.19, n.3, p.326-332, 1999.

OLIVEIRA, M.E.B. et al. Perfil químico de qualidade das polpas de acerola, cajá e caju comercializadas no estado da Bahia. Revista Brasileira de Fruticultura, v.22, Número especial, p.9-15, 2000.

QUEIROZ, J.A.L. Produção de mudas de taperebá. Macapá: Embrapa Amapá, 2000. 3p. (Embrapa Amapá. Recomendações técnicas, 11).

SAEG. Sistema para análises estatísticas. Versão 9.0 Viçosa: Fundação Arthur Bernardes, 2005.

SILVA, A.P.V. Processamento e estabilidade dos sucos polposo e clarificado de cajá (Spondias lutea L.). 1995 116f. Dissertação (Mestrado em Tecnologia de alimentos) Faculdade de Engenharia de Alimentos, Universidade Federal do Ceará.

SILVA, A.P.V. et al. Estudo da produção do suco clarificado de cajá (Spondias lutea L.). Ciência e Tecnologia de Alimentos, v.19, n.1, p.326-332, 1999.

SILVA, M. et al. Resfriamento e propriedades termofísicas do cajá (Spondias lutea L.). Revista Brasileira de Produtos Agroindustriais, Campina Grande, v.4, n.2, p.175-185, 2002.

SILVA, A.P.V. et al. Características de qualidade do suco polposo de cajá (Spondias lútea L.) obtido por extração mecânicoenzimática. Ciência e Tecnologia de Alimentos, Campinas, v.17, n.3, p.233-236, 1997 .

SOUZA FILHO, M.F. et al. Manejo integrado de pragas na cultura da manga. In: ROZANG, D.E. et al. (Ed.). Manga Produção integrada, industrialização e comercialização. Viçosa: UFV, 2004. p.339-376.

VIEIRA NETO, R.D. Fruteiras potenciais para os tabuleiros costeiros e baixadas litorâneas. Aracajú: Embrapa Tabuleiros Costeiros/Empresa de Desenvolvimento Agropecuário de Sergipe - Emdagro, 2002. 216p. 